\title{
High temperature quark localization by Polyakov loops
}

\author{
Tamás G. Kovács ${ }^{* \dagger}$ and Ferenc Pittler ${ }^{\dagger}$ \\ Department of Physics, University of Pécs \\ H-7624 Pécs, Ifjúság útja 6, Hungary
}

\section{Falk Bruckmann ${ }^{\ddagger}$ and Sebastian Schierenberg \\ Institut für Theoretische Physik \\ D-93040 Regensburg, Germany}

\begin{abstract}
We study the low eigenmodes of the overlap and staggered Dirac operator at high temperature. We show that the recently found localized quark modes obeying Poisson statistics are connected to physical gauge field objects with their size and density scaling in the continuum limit. The localized modes are also strongly correlated with large fluctuations of the Polyakov loop. Based on that we construct a random matrix model of the low Dirac modes inspired by dimensional reduction. Our model reproduces the Poisson to random matrix transition seen in the lattice Dirac spectrum.
\end{abstract}

The XXIX International Symposium on Lattice Field Theory - Lattice 2011

July 10-16, 2011

Squaw Valley, Lake Tahoe, California

\footnotetext{
* Speaker.

${ }^{\dagger}$ Supported by EU Grant (FP7/2007-2013)/ERC N²08740. We thank the Budapest-Wuppertal collaboration for providing us their code for generating the dynamical configurations used in this work.

${ }^{\ddagger}$ Supported by DFG Grant (BR-2872/4-2).
} 


\section{Introduction}

It is by now well established that the high temperature QCD Dirac spectrum has a remarkable feature, a transition from localized to delocalized modes [1]-[3]. It seems to be a generic feature of non-Abelian gauge theories in four dimensions that at high temperature, where chiral symmetry is restored, the lowest part of the Dirac spectrum consists of localized modes exhibiting Poisson statistics. Higher up in the spectrum there is a cross-over to delocalized modes and random matrix statistics persisting throughout the bulk of the spectrum. This behavior, reminiscent of Anderson localization, was first seen in the quenched $S U(2)$ theory with the overlap Dirac operator [2] and later with the staggered Dirac operator [3].

The detailed mechanism behind quark localization is still not known. In particular, it is not clear whether there are any easily identifiable gauge field configurations that are capable of binding and localizing the lowest quark eigenmodes. In the present paper, which is mostly based on Ref. [4], we present some new findings concerning the nature of these gauge field objects. The lattice data we use in support of these results come from various simulations including overlap and staggered Dirac spectra in quenched $S U(2)$ gauge backgrounds and stout smeared staggered spectra in $S U(3)$ backgrounds with $2+1$ flavors of dynamical quarks. The results of the dynamical simulations have not been published yet, some preliminary results appear in the presentation by F. Pittler at this Conference.

\section{Correlation between overlap and staggered modes}

If the low Dirac modes are localized on specific gauge objects then in a given gauge field background one expects the localized modes to occur around similar locations irrespective of the particular discretization of the Dirac operator. To test this we compared the lowest twelve eigenmodes of the staggered and the overlap Dirac operator [6] on the same set of gauge field backgrounds. For quantifying the spatial overlap between two eigenmodes we defined the quantity

$$
I=V \sum_{x}\left|\phi_{\text {stag }}(x)\right|^{2}\left|\psi_{\mathrm{ov}}(x)\right|^{2}
$$

that we call interlocalization. For non-overlapping modes $I$ can be close to zero while for exactly identical modes $I$ coincides with the inverse of the participation ratio. Here the eigenmodes are always assumed to be normalized. To have a meaningful comparison we first paired the lowest overlap eigenmode with the staggered mode that had the largest interlocalization with it. Then the second lowest overlap mode was paired with the remaining staggered mode with maximal interlocalization with it and so on. After this pairing was done configuration by configuration, we computed the average interlocalization for the lowest twelve overlap modes and the corresponding staggered modes. In Figure 1 we plot this quantity as a function of the overlap eigenvalue both in the $Q=0$ and the $|Q|=1$ topological sectors.

Indeed, in both topological sectors the lowest staggered and overlap eigenmodes have substantial overlaps that diminishes higher up in the spectrum. We note that putting Gaussian random amplitudes for the two vectors yields an interlocalization of unity. We can thus conclude that there are some objects in the gauge configurations that bind localized eigenmodes of both the overlap 


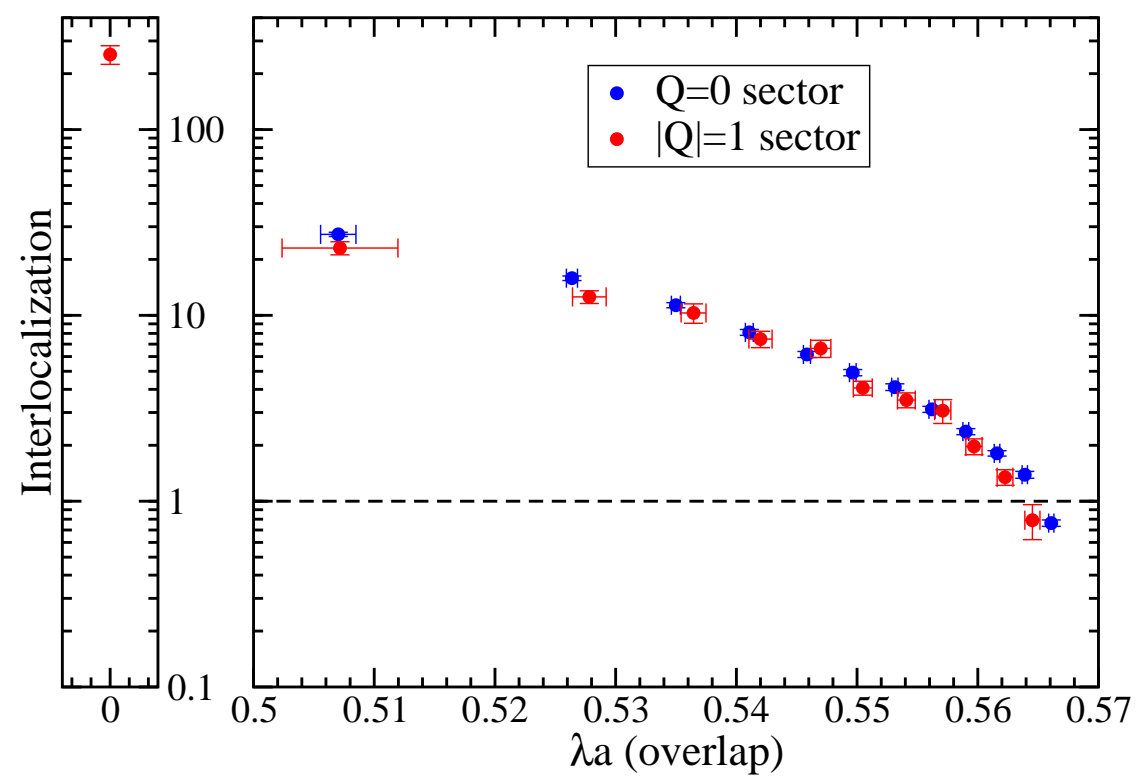

Figure 1: The average interlocalization (see Eq. (2.1)) of the lowest overlap and staggered eigenmodes as a function of the overlap eigenvalue in the topological charge sectors $Q=0$ and $|Q|=1$

and the staggered Dirac operators. We note here that similarities between overlap and staggered spectra had already been noted for the Schwinger model [7] and for QCD [8], but in our case the similarity also extends to the spatial structure of the eigenmodes.

\section{Scaling in the continuum limit}

Since the lowest eigenmodes are localized to within a few lattice spacings one might be inclined to identify these gauge field objects with some sort of "dislocations", unusually large fluctuations in the gauge fields on the scale of the lattice spacing. To explore this possibility we repeated the simulations on three finer grids and, based on the participation ratio, computed the linear size of the localized eigenmodes [5]. All these simulations were done at the same physical temperature $\left(T=2.6 T_{c}\right)$ and in spatial boxes of the same physical size with the lattice spacing set by the critical temperature. The results are shown in Figure 2 where we plot the eigenvalue size in units of the inverse temperature. A priori it is not clear how to extrapolate this quantity to the continuum limit, but to guide the eye we included a linear extrapolation. It is obvious that using any sensible extrapolation to the continuum limit yields a non-zero value, in fact a number of order unity. This clearly rules out dislocations as candidates for binding the localized modes. Moreover, it seems that the spatial size of the localized modes is set by the box size in the temporal direction.

To obtain further information concerning the nature of the gauge objects binding the localized modes we can also look at their density and in particular how that scales in the continuum limit. Since there is no sharp boundary between the localized and the delocalized modes in the spectrum, it is not straightforward to count the number of localized modes and to define their density. For instance in terms of the level spacing distribution, $P(s)$, the exponential distribution, $P(s)=\exp (-s)$, 


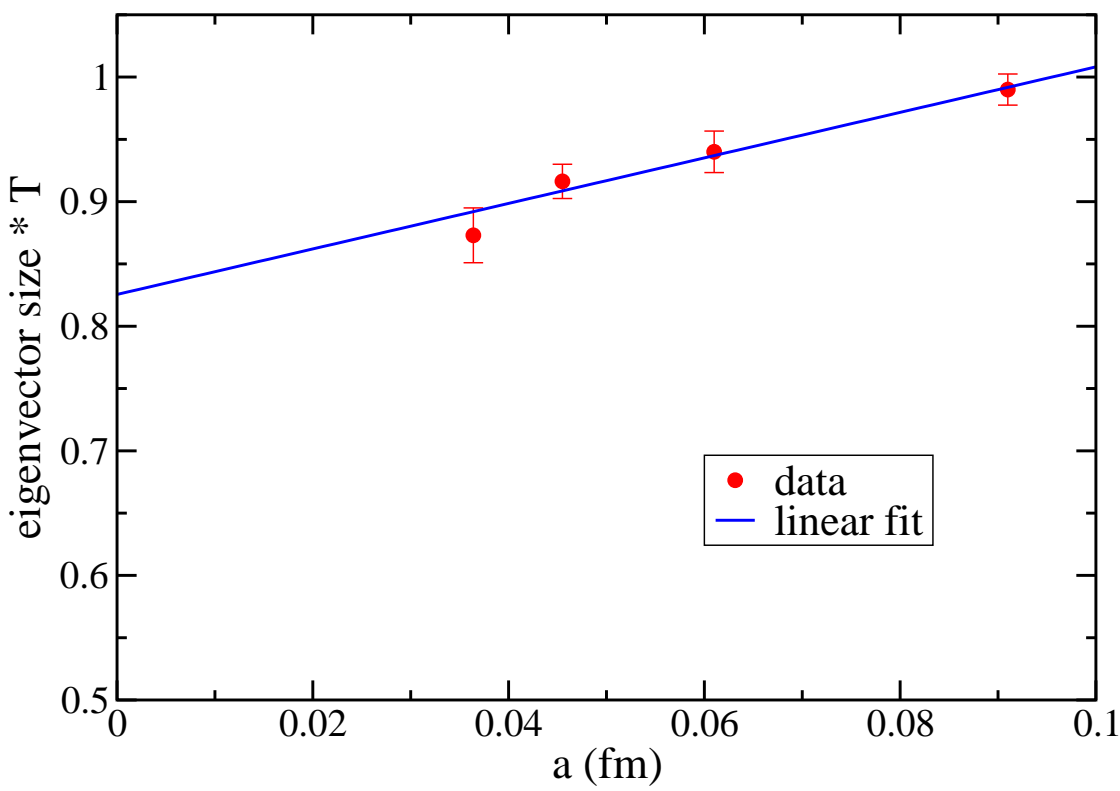

Figure 2: The average linear size of the localized low eigenmodes of the overlap Dirac operator as a function of the lattice spacing. The eigenmode size is measured in units if inverse temperature.

characterizing localized modes continuously changes into the Wigner surmise as we go up in the spectrum and reach the regime of delocalized modes. If this path of deformation is universal, which seems to be the case here, one can arbitrarily choose a "standard" distribution somewhere between the exponential and the Wigner surmise. One can then call those eigenmodes localized that are below the point in the spectrum where the level spacing distribution reaches this standard "in-between" distribution.

In Figure 3 we show the unfolded level spacing distribution computed from eigenvalues between number 10 and 20 of the staggered Dirac operator in gauge field backgrounds generated with $2+1$ flavors of dynamical fermions with physical masses using the action in Ref. [9]. The three curves correspond to three different ensembles with $N_{t}=4,6$ and 8 , at the same physical temperature and with the same physical spatial box size. The two curved lines indicate the exponential and the unitary Wigner surmise distributions observed in the lowest part and the bulk of the spectrum. The histograms are exactly on top of one another illustrating that the deformation of the distribution occurs along the same universal path regardless of the lattice spacing. Since the matching histograms correspond to the same slice of the spectrum (between eigenvalues 10 and 20) in all three cases, we can conclude that according to our definition all these ensembles have the same number of localized modes per configuration. This in turn implies that the physical density of localized modes is also the same since the physical three-volumes of the ensembles were chosen to be identical.

We already know that localized low Dirac modes are bound to some gauge objects that have a fixed physical size and physical density in the continuum limit. It would be tempting to identify these as finite temperature topological objects, calorons with their constituent monopoles. Based on the zero modes of the overlap Dirac operator on these gauge backgrounds we can estimate that 


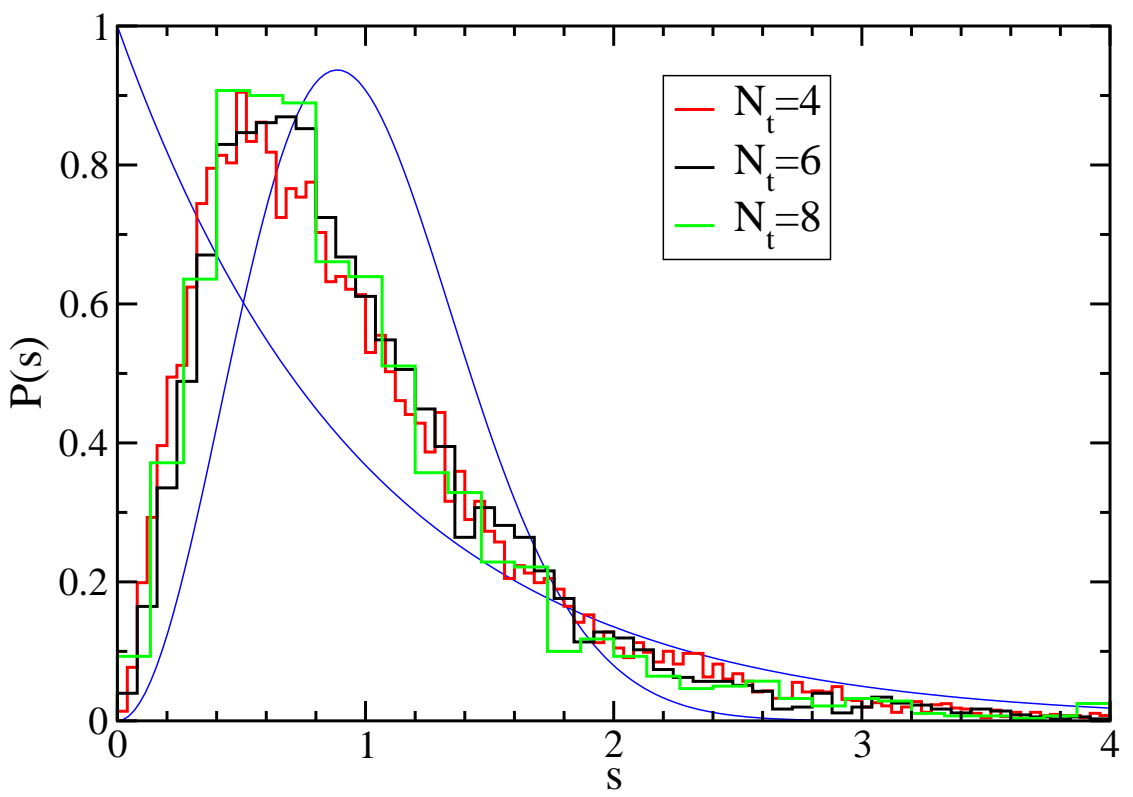

Figure 3: The unfolded level spacing distribution computed from the eigenvalues between the $10^{\text {th }}$ and $20^{\text {th }}$. The histograms correspond to three ensembles with the same physical parameters, differing only in the lattice spacing. The two curved lines indicate the exponential and unitary Wigner surmise distributions corresponding to the lowest part and the bulk of the spectrum.

at this temperature there are on average about 0.01 topological objects (calorons or anti-calorons) per cubic fermi. This estimate also depends on the assumption that since the gas of topological objects is dilute, they are uncorrelated. On the other hand, the density of localized modes turns out to be about 1 per cubic fermi. The two orders of magnitude discrepancy between the density of calorons and localized modes makes it impossible that a dilute gas of topological objects can explain the localized Dirac modes.

\section{Connection to Polyakov loops}

To gain further intuition concerning the origin of these low Dirac modes we note that the "thinning out" of Dirac modes around zero at high temperature, needed for chiral restoration, can be qualitatively understood by considering the lowest Matsubara frequency. Indeed, due to the anti-periodic temporal boundary condition the lowest free quark modes are shifted away from zero by an amount $\propto T$. If the gauge field is "turned on" the effective temporal boundary condition for the quarks is the combination of the anti-periodic boundary condition and the Polyakov loop. At high temperature the latter has a positive real expectation value, however, it can locally fluctuate. Fluctuations of the Polyakov loop can in principle locally lower the effective Matsubara frequency and shift some Dirac eigenvalues towards zero. If this is the case one expects the lowest eigenmodes to be localized at places where the Polyakov loop has large fluctuations.

Whether that is the case can be tested by comparing the weighted average of the Polyakov loop to the simple average. We choose the weight on each site to be the magnitude of a normalized 


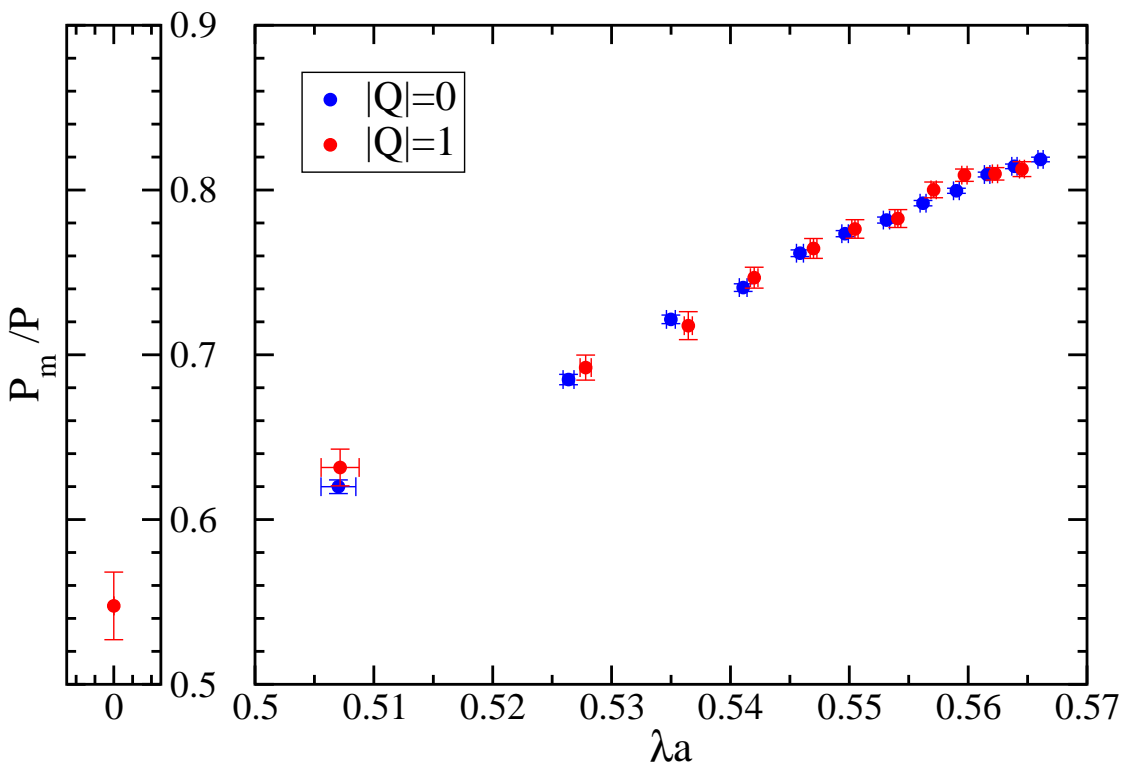

Figure 4: The weighted average of the Polyakov loop versus the overlap eigenmode the magnitude of which was used for the weighting. The weighted average is normalized by the simple average.

Dirac wave function on that site. In this way to each Dirac eigenmode we can associate a weighted Polyakov loop with the formula

$$
P_{m}=\sum_{x} P(x)\left|\psi_{m}(x)\right|^{2}
$$

In Figure 4 we plot this quantity normalized by the simple average Polyakov loop for the pure $S U(2)$ gauge theory for the first few overlap eigenvalues. It is clear that for the low Dirac modes the weighted average is significantly lower than the simple average. This demonstrates that the localized Dirac modes are indeed peaked at locations where the Polyakov loop has large local fluctuations towards smaller and even negative values that significantly lower the effective Matsubara frequency.

To test the validity of this picture in a more quantitative fashion we also explored an effective random matrix model inspired by this picture. Splitting the Dirac operator into a temporal and a spatial part we choose a basis that diagonalizes the temporal part with diagonal matrix elements

$$
\lambda(x) a=\sin \frac{\pi-\phi(x)}{N_{t}}
$$

where $\lambda(x)$ is the effective local lattice Matsubara frequency and $\phi(x)$ corresponds to the local phase of the Polyakov loop at $x$. The extra phase $\pi$ in this formula represents the anti-periodic temporal boundary condition. We further assume that this three-dimensional array of local Matsubara modes interact through nearest neighbor interactions in all spatial directions. These random spatial interactions are meant to capture the effect of spatial gauge couplings in the Dirac operator. This sparse random matrix model has a dimensionally reduced three-dimensional structure and is analogous to the Anderson model based on the tight binding approximation. The random on-site terms are the local Matsubara frequencies and the nearest neighbor hopping terms are the effective 
interaction terms between neighboring Matsubara modes. This model has only a few parameters to be fixed. We experimented with some parameter sets inspired by actual lattice data and found that for a range of parameters this random matrix ensemble reproduces the qualitative features of the lattice Dirac operator. The lowest part of the spectrum consists of localized modes with exponentially distributed nearest neighbor level spacings and the bulk of the spectrum has delocalized modes with random matrix statistics. For details we refer the reader to Ref. [4].

\section{Conclusions}

In the present paper we studied how the localization of the lowest quark modes occurs in QCD at high temperature. We showed that the location of the eigenmodes is robust with respect to different discretizations of the Dirac operator. We demonstrated this by comparing staggered and overlap eigenmodes on the same gauge backgrounds. Using simulations with four different lattice spacings we found that the physical size of the localized modes has a non-zero continuum limit which is of the order of the inverse temperature. This implies that localized modes are not bound to dislocations, rather they are connected to gauge field objects the extension of which is controlled by the temporal box size. We further demonstrated that the physical density of these objects does not depend on the lattice spacing. Their density turned out to be about two orders of magnitude larger than the density of topological objects. We found strong correlations between the locations of low Dirac modes and those of large fluctuations of the Polyakov loop. Based on that we proposed a dimensionally reduced sparse random matrix model of localization. It would be interesting to explicitly identify the gauge field objects responsible for localization. Monopoles and dyons are good candidates for that, but some non-trivial correlations between different objects would be needed [10],[11].

\section{References}

[1] A. M. Garcia-Garcia and J. C. Osborn, Phys. Rev. D 75, 034503 (2007) [arXiv:hep-lat/0611019]; A. M. Garcia-Garcia, J. C. Osborn, Nucl. Phys. A770, 141-161 (2006). [hep-lat/0512025].

[2] T. G. Kovacs, Phys. Rev. Lett. 104, 031601 (2010) [arXiv:0906.5373 [hep-lat]].

[3] T. G. Kovacs, F. Pittler, Phys. Rev. Lett. 105, 192001 (2010). [arXiv:1006.1205 [hep-lat]].

[4] F. Bruckmann, T. G. Kovacs, S. Schierenberg, Phys. Rev. D84, 034505 (2011). [arXiv:1105.5336 [hep-lat]].

[5] T. G. Kovacs, F. Pittler, PoS LATTICE2010, 195 (2010). [arXiv:1011.3175 [hep-lat]].

[6] H. Neuberger, Phys. Lett. B417, 141 (1998), hep-lat/9707022; H. Neuberger, Phys. Lett. B427, 353 (1998), hep-lat/9801031].

[7] S. Dürr, C. Hoelbling, Phys. Rev. D69, 034503 (2004), hep-lat/0311002.

[8] S. Dürr, C. Hoelbling, U. Wenger, Phys. Rev. D70, 094502 (2004), hep-lat/0406027.

[9] Y. Aoki, Z. Fodor, S. D. Katz, K. K. Szabo, Phys. Lett. B643, 46-54 (2006). [hep-lat/0609068].

[10] E. -M. Ilgenfritz, E. V. Shuryak, Phys. Lett. B325, 263-266 (1994). [hep-ph/9401285].

[11] M. Unsal, Phys. Rev. D 80, 065001 (2009) [arXiv:0709.3269 [hep-th]]. 